\title{
OPEN Multipartite entanglement criterion via generalized local uncertainty relations
}

\author{
Jia-Bin Zhang ${ }^{1}$, Tao $\mathrm{Li}^{2 \bowtie}$, Qing-Hua Zhang ${ }^{1}$, Shao-Ming Fei ${ }^{1}$ \& Zhi-Xi Wang ${ }^{1}$
}

We study the detection of multipartite entanglement based on the generalized local uncertainty relations. A sufficient criterion for the entanglement of four-partite quantum systems is presented in terms of the local uncertainty relations. Detailed examples are given to illustrate the advantages of our criterion. The approach is generalized to general multipartite entanglement cases.

Quantum entanglement is a remarkable feature in quantum physics ${ }^{1}$ and has attracted much attention in recent years. Entangled states are recognized as the essential resources in quantum information processing, with many experimental realizations $s^{2,3}$ and applications in such as quantum algorithms ${ }^{4}$, quantum teleportation ${ }^{4,5}$, quantum cryptography ${ }^{6}$. Recently, it was shown that quantum entanglement is tightly connected to wave-particle duality, and it can create a wave-particle entangled state of two photons ${ }^{7}$. Detecting entanglement of multipartite systems is a fundamental problem in the theory of quantum entanglement. Separability criteria to determine whether a given state is separable or not are of crucial importance ${ }^{8}$. Enormous efforts have been dedicated to solve the separability problems ${ }^{9-37}$. Nevertheless, the characterization and quantification of multipartite entanglement are less understood than that of bipartite case, as multipartite states can be entangled in more different ways.

There have been many efficient entanglement criteria such as local uncertainty relations (LUR) ${ }^{11,12}$, covariance matrix criterion $(\mathrm{CMC})^{13}$, computable cross-norm or realignment criterion $(\mathrm{CCNR})^{14}$, permutation separability criteria $^{15}$, criterion based on Bloch representations ${ }^{17,18}$, entanglement witnesses ${ }^{21}$, Bell-type inequalities criteria ${ }^{22}$, and criterion based on quantum Fisher information ${ }^{23}$. Generally, these criteria are only necessary condition for separable states and have different advantages in detect different entanglements.

The LUR criterion, the symmetric CMC criterion and the realignment criterion are usually considered as complementary to the the positive partial transposition criterion. The main advantage of LUR criterion is that it allows us to detect the entanglement of quantum states without having to fully understand them, and it can detect bound entangled states more effectively.

Recently, based on the local sum uncertainty relations, some entanglement criteria have been proposed for both discrete and continuous variable bipartite systems and three-qubit systems ${ }^{31-33}$. Zhang et al. proposed a tighter form of the original LUR criterion to improve the range of entanglement detection ${ }^{31}$, Akbari-Kourbolagh and Azhdargalam generalized the LUR criterion to the tripartite systems ${ }^{33}$.

This paper is structured as follows. We start by introducing the entanglement criterion based on LUR for tripartite systems and generalize the entanglement criterion to four-partite quantum systems. Some detail examples are then given to illustrate the advantages of the criterion. Then, the entanglement criterion for $N$-partite systems $(N>4)$ is discussed. Brief discussion and summary are given at last.

\section{Results}

Let $\mathcal{H}=\mathcal{H}_{1} \otimes \mathcal{H}_{2} \otimes \cdots \otimes \mathcal{H}_{N}$ be an $N$-partite system with $\mathcal{H}_{k}$ the $d_{k}$-dimensional vector space associated with the $k$-th subsystem. An $N$-partite state $\rho \in \mathcal{H}$ is said to be separable if $\rho$ can be written as

$$
\rho=\sum_{i} p_{i} \rho_{i}^{1} \otimes \rho_{i}^{2} \otimes \cdots \otimes \rho_{i}^{N}
$$

where $\rho_{i}^{k}$ are density matrices of the subsystem $\mathcal{H}_{k}, 0 \leq p_{i} \leq 1, \sum_{i} p_{i}=1$.

In quantum theory, the observables of a quantum system are represented by a set of Hermitian operators $\left\{A_{i}\right\}$. The uncertainty principle shows that it is impossible to predict the measurement results of all observables of the system at the same time. The variance of $A_{i}$ with respect to $\rho$ is the uncertainty of an observable $A_{i}$, defining as $\left(\Delta A_{i}\right)_{\rho}^{2}=\left\langle A_{i}^{2}\right\rangle_{\rho}-\left\langle A_{i}\right\rangle_{\rho}^{2}$, where $\left\langle A_{i}\right\rangle_{\rho}=\operatorname{Tr}\left(\rho A_{i}\right)$ is the mean value. For a set of quantum observables $\left\{A_{i}\right\}$, there 
exits a constant $U$ such that $\sum_{i}\left(\Delta A_{i}\right)_{\rho}^{2} \geq U$. This inequality gives a universally valid limitation of the measurement outcomes. Generally, it is difficult to determine the value $U$. For the case of Pauli matrices $\sigma_{x}, \sigma_{y}$ and $\sigma_{z}$, one has $\left(\Delta \sigma_{x}\right)_{\rho}^{2}+\left(\Delta \sigma_{y}\right)_{\rho}^{2}+\left(\Delta \sigma_{z}\right)_{\rho}^{2} \geq 2^{32}$.

In Ref. ${ }^{33}$, based on the local sum uncertainty relations, an entanglement criterion has been presented for tripartite systems.

Let $\left\{A_{1}^{i}\right\},\left\{A_{2}^{i}\right\}$ and $\left\{A_{3}^{i}\right\}$ be the set of local observables associated to the subsystems $\mathcal{H}_{1}, \mathcal{H}_{2}$ and $\mathcal{H}_{3}$, respectively. $U_{1}, U_{2}, U_{3}$ are lower bound of these local observables, such that $\sum_{i} \Delta\left(A_{2}^{i}\right)^{2} \geq U_{1}, \sum_{i} \Delta\left(A_{2}^{i}\right)^{2} \geq U_{2}$ and $\sum_{i} \Delta\left(A_{3}^{i}\right)^{2} \geq U_{3}$. For any separable tripartite states, the following inequalities hold under any permutations of $\{1,2,3\}^{33}$ :

$$
F_{\rho}^{12 \mid 3} \equiv \sum_{i} \Delta\left(A_{1}^{i}+A_{2}^{i}+A_{3}^{i}\right)_{\rho}^{2}-\left(U_{1}+U_{2}+U_{3}+M_{12}^{2}+M_{12 \mid 3}^{2}\right) \geq 0
$$

wh e re $\quad M_{12}=\sqrt{\sum_{i} \Delta\left(A_{1}^{i}\right)^{2}-U_{1}}-\sqrt{\sum_{i} \Delta\left(A_{2}^{i}\right)^{2}-U_{2}}, \quad M_{12 \mid 3}=\sqrt{F_{\rho}^{12}}-\sqrt{\sum_{i} \Delta\left(A_{3}^{i}\right)^{2}-U_{3}}$, $F_{\rho}^{12}=\sum_{i} \Delta\left(A_{1}^{i}+A_{2}^{i}\right)^{2}-\left(U_{1}+U_{2}+M_{12}^{2}\right), A_{1}^{i}, A_{2}^{i}$ and $A_{3}^{i}$ are the operators acting on the first, the second and the third subsystem with the rest subsystems as identity operators in the tripartite systems, respectively.

Generalizing the criterion (2) to four-partite systems, we consider the set of local observables $\left\{A_{1}^{i}\right\},\left\{A_{2}^{i}\right\},\left\{A_{3}^{i}\right\}$ and $\left\{A_{4}^{i}\right\}$ associated to the subsystems $\mathcal{H}_{1}, \mathcal{H}_{2}, \mathcal{H}_{3}$ and $\mathcal{H}_{4}$, respectively. From the local sum uncertainty relations, there must exists lower bounds $U_{j}>0$ for each nonsimultaneous observable $\left\{A_{j}^{i}\right\}$ for $j=1,2,3,4$. That is to say,

$$
\sum_{i} \Delta\left(A_{2}^{i}\right)^{2} \geq U_{1}, \quad \sum_{i} \Delta\left(A_{2}^{i}\right)^{2} \geq U_{2}, \quad \sum_{i} \Delta\left(A_{3}^{i}\right)^{2} \geq U_{3}, \quad \sum_{i} \Delta\left(A_{4}^{i}\right)^{2} \geq U_{4} .
$$

Then for four-partite quantum systems, we have the following conclusion.

Theorem 1 For any four-partite separable states, the following inequalities hold simultaneously under any permutations of $\{1,2,3,4\}$,

$$
\begin{aligned}
& F_{\rho}^{123 \mid 4}=F-\left(M_{12}^{2}+M_{12 \mid 3}^{2}+M_{123 \mid 4}^{2}\right) \geq 0, \\
& F_{\rho}^{12 \mid 34}=F-\left(M_{12}^{2}+M_{34}^{2}+M_{12 \mid 34}^{2}\right) \geq 0,
\end{aligned}
$$

where $\quad F=\sum_{i} \Delta\left(A_{1}^{i}+A_{2}^{i}+A_{3}^{i}+A_{4}^{i}\right)_{\rho}^{2}-\sum_{j=1}^{4} U_{j}, \quad M_{123 \mid 4}=\sqrt{F_{\rho}^{12 \mid 3}}-\sqrt{\sum_{i} \Delta\left(A_{4}^{i}\right)^{2}-U_{4}}$, $M_{12 \mid 34}=\sqrt{F_{\rho}^{12}}-\sqrt{F_{\rho}^{34}}$.

Theorem 1 provides a necessary condition of separable four-partite states. The violations of the inequalities in (1) sufficiently imply entanglement. For the four-qubit $W$ state, $\rho=\left|W_{4}\right\rangle\left\langle W_{4}\right|$ with $\left|W_{4}\right\rangle=\frac{1}{2}(|1000\rangle+|0100\rangle+|0010\rangle+|0001\rangle)$. Let $A_{1}^{1}=A_{2}^{1}=A_{3}^{1}=-A_{4}^{1}=\sigma_{x}, A_{1}^{2}=A_{2}^{2}=A_{3}^{2}=-A_{4}^{2}=\sigma_{y}$ and $A_{1}^{3}=A_{2}^{3}=A_{3}^{3}=-A_{4}^{3}=\sigma_{z}$, thus we get $\sum_{i} \Delta\left(A_{j}^{i}\right)^{2} \geq 2, M_{12}=0, M_{34}=0, M_{12 \mid 3}=\sqrt{3}-\sqrt{\frac{3}{4}}$, $M_{123 \mid 4}=\sqrt{\frac{27}{4}-M_{12 \mid 3}^{2}}-\sqrt{\frac{3}{4}}$ and $M_{12 \mid 34}=\sqrt{3}$, which give rise to $F_{\rho}^{123 \mid 4}=3-M_{12 \mid 3}^{2}-M_{123 \mid 4}^{2}<0$ and $F_{\rho}^{12 \mid 34}=0$, which provide a violation for the inequalities (4). Therefore, the criterion identifies four-qubit $W$ state is entangled. By taking use of Theorem 1, more generally states can be detected and we consider some detailed examples for mixed states below.

Example 1 (Four-qubit $W$ state mixed with white noise) We first consider $\rho_{1}=\frac{p}{16} I+(1-p)\left|W_{4}\right\rangle\left\langle W_{4}\right|$, $0 \leq p \leq 1$. For this state, we choose $-A_{1}^{1}=-A_{2}^{1}=-A_{3}^{1}=A_{4}^{1}=\sigma_{x},-A_{1}^{2}=-A_{2}^{2}=A_{3}^{2}=A_{4}^{2}=\sigma_{y}$ and $-A_{1}^{3}=-A_{2}^{3}=-A_{3}^{3}=-A_{4}^{3}=\sigma_{z}$, hence $\sum \Delta\left(A_{j}^{i}\right)^{2} \geq 2, M_{12}=M_{34}=0, M_{12 \mid 3}=\sqrt{3-p^{2}}-\sqrt{1-\frac{1}{4}(1-p)^{2}}$, $M_{123 \mid 4}=\sqrt{\frac{10 p-9 p^{2}+11}{4}-M_{12 \mid 3}^{2}}-\sqrt{1-\frac{1}{4}(1-p)^{2}}$ and $M_{12 \mid 34}=\sqrt{3-p^{2}}-\sqrt{2 p-p^{2}+1}$. Then, we get $F_{\rho_{1}}^{123 \mid 4}=10 p-4 p^{2}-2-M_{12 \mid 3}^{2}-M_{123 \mid 4}^{2}$ and $F_{\rho_{1}}^{12 \mid 34}=10 p-4 p^{2}-2-M_{12 \mid 34}^{2}$. When $p \leq 0.3605, F_{\rho_{1}}^{123 \mid 4} \leq 0$, so the state $\rho_{1}$ violates one of the inequalities (4). Therefore, the four-partite LUR criterion identifies the $\rho_{1}$ as an entangled state, see Fig. 1 . While, $\rho_{1}$ is detected based on the witness $\mathcal{W}=\frac{3}{4} I-\left|W_{4}\right\rangle\left\langle W_{4}\right|$ which is proposed in Ref. ${ }^{27}$ when $p<0.267$, see Fig. 2 . That is to say our result detects better the entanglement than the criterion of Ref. ${ }^{27}$. 


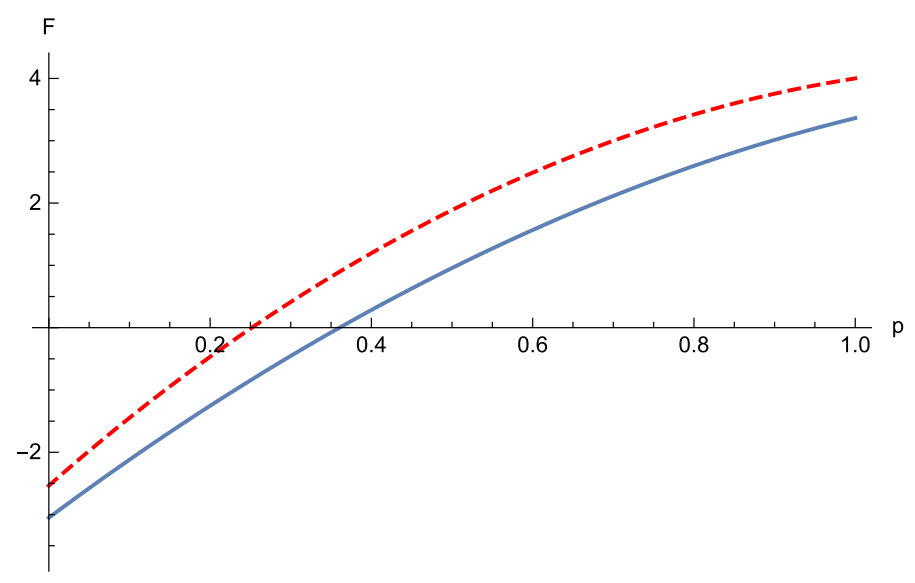

Figure 1. For the four-partite $W$ state mixed with the white noise $\rho_{1}$. The the blue line stands for $F_{\rho_{1}}^{123 \mid 4}$ and the red dash line stands for $F_{\rho_{1}}^{12 \mid 34}$ in Theorem 1 . We can see that when $p \leq 0.3605$, state $\rho_{1}$ violates one of the inequalities (4), hence $\rho_{1}$ is entangled for $p \leq 0.3605$.

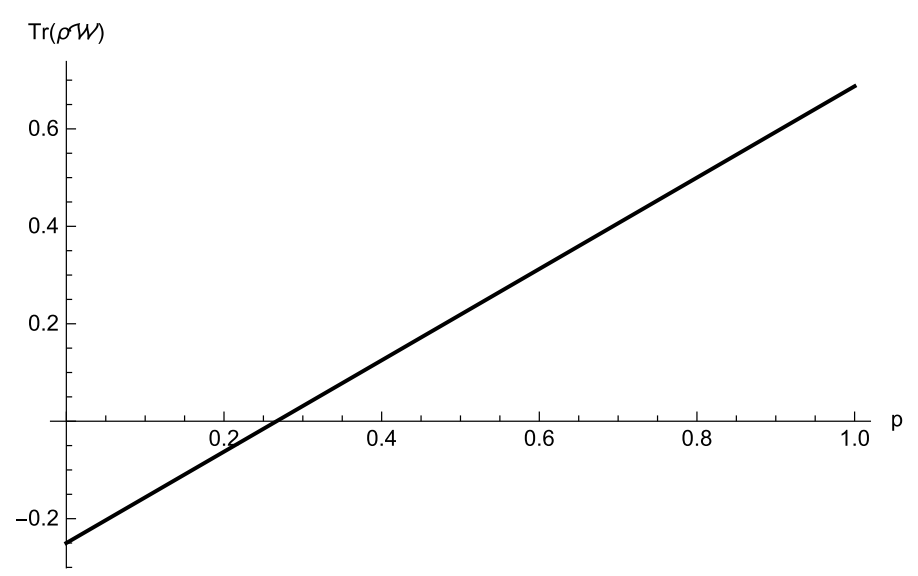

Figure 2. For the four-partite $W$ state mixed with the white noise $\rho_{1}$. The the black line represents $\operatorname{Tr}\left(\rho_{1} \mathcal{W}\right)$ in Ref. ${ }^{27}$. We can see that $\rho_{1}$ is detected by the witness $\frac{3}{4} I-\left|W_{4}\right\rangle\left\langle W_{4}\right|$, thus $\rho_{1}$ is entangled for $p \leq 0.267$.

Example 2 (Four-qubit Dicke state mixed with white noise) Now, we take $\rho_{2}=\frac{p}{16} I+(1-p)\left(\left|D_{2}^{4}\right\rangle\left\langle D_{2}^{4}\right|\right)$, $0 \leq p \leq 1$, where $\left|D_{2}^{4}\right\rangle=\frac{1}{\sqrt{6}}(|1100\rangle+|1010\rangle+|1001\rangle+|0110\rangle+|0101\rangle+|0011\rangle)$. For this state, we choose $-A_{1}^{1}=-A_{2}^{1}=A_{3}^{1}=A_{4}^{1}=\sigma_{x}, A_{1}^{2}=A_{2}^{2}=A_{3}^{2}=-A_{4}^{2}=\sigma_{y},-A_{1}^{3}=-A_{2}^{3}=-A_{3}^{3}=-A_{4}^{3}=\sigma_{z}$. By direct calculations, we get $M_{12}=0, M_{34}=0, M_{12 \mid 3}=\sqrt{4-2 p}-1, M_{123 \mid 4}=\sqrt{\frac{35}{3}-\frac{26}{3} p-M_{123}^{2}}-1$ and $M_{12 \mid 34}=\sqrt{4-2 p}-\sqrt{2 p}, \quad$ which $\quad$ yield $\quad F_{\rho_{2}}^{123 \mid 4}=\frac{22}{3}(p-1)+\frac{2 \sqrt{6}}{3} \sqrt{2 p-2+3 \sqrt{4-2 p}} \quad$ and $F_{\rho_{2}}^{12 \mid 34}=8 p-8+4 \sqrt{\frac{4-p^{2}}{3}}$. When $p \leq 0.437, F_{\rho_{2}}^{12 \mid 34} \leq 0$, and $F_{\rho_{2}}^{123 \mid 4} \leq 0$ for $p \leq 0.543$. It can be seen, from Fig. 3 , that the $\rho_{2}$ violate inequalities (4) for $p \leq 0.543$. Furthermore, comparing with the result in Ref. ${ }^{27}$ which show that $\rho_{2}$ is entangled for $p<0.356$ (see Fig. 4), the Theorem 1 also detects more entanglement.

For a more general case, we consider the set of local observable $\left\{A_{1}^{i}\right\},\left\{A_{2}^{i}\right\}, \ldots,\left\{A_{N}^{i}\right\}$ associated to the subsystems $\mathcal{H}_{1}, \mathcal{H}_{2}, \ldots, \mathcal{H}_{N}$, respectively. Every local observable has a lower bound $U_{j}(j=1,2, \ldots, N)$ satisfies $\sum\left(A_{j}^{i}\right)^{2} \geq U_{j}$. In order to simplify calculation, let $i_{N}$ represent $\left\{A_{i_{N}}^{i}\right\}$ and the bi-partition index $\left(i_{1} i_{2} \cdots i_{K} \mid i_{K+1} \cdots i_{N}\right)$ is denoted as $k_{1} \mid k_{0}$, where $k_{1}=i_{1} i_{2} \cdots i_{K}$ and $k_{0}=i_{K+1} i_{K+2} \cdots i_{N},\left\lceil\frac{N}{2}\right\rceil \leq K<N$ and $1 \leq i_{1}<i_{2}<\cdots<i_{K} \leq N$. For instance, if $N=4$, hence $K=2$, and $k_{1} \mid k_{0}=\{12|34,13| 24,14 \mid 23\}$, which represents three classes of bi-partition index of local observable set in $\mathrm{N}$-body quantum system. Similar to the derivation of the Theorem 1, we obtain the following lemma and theorem.

Lemma 2 For multipartite separable states, the following inequalities must hold: 


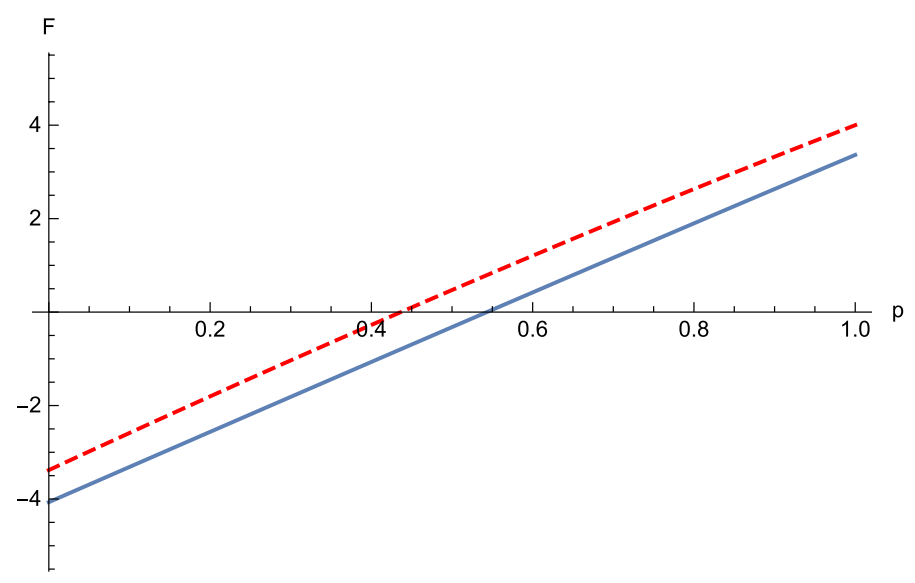

Figure 3. For the four-partite Dicke state $D_{2}^{4}$ mixed with the white noise $\rho_{2}$. The the blue line stands for $F_{\rho_{2}}^{123 / 4}$ and the red dash line stands for $F_{\rho_{2}}^{123 \mid 4}$ and the red dash line stands for $F_{\rho_{2}}^{12 \mid 34}$ in Theorem 1 . When $p \leq 0.3605$, we can see that the state $\rho_{2}$ violates one of the inequalities (4), whence our criterion detects the entanglement of $\rho_{2}$ for $0 \leq p \leq 0.543$.

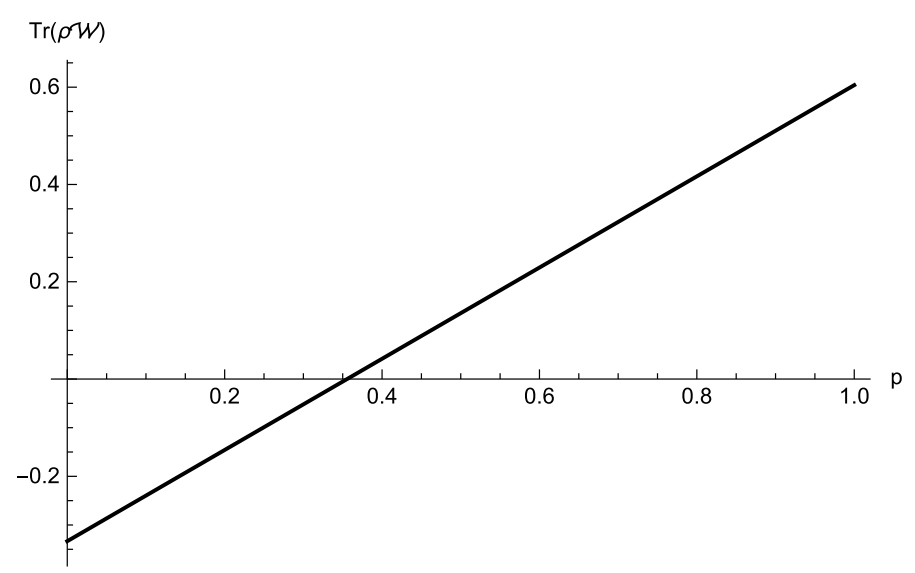

Figure 4. For the four-partite Dicke state $D_{2}^{4}$ mixed with the white noise $\rho_{2}$. The the black line stands for $\operatorname{Tr}(\rho \mathcal{W})$ in Ref. ${ }^{27}$. By using the witness $\mathcal{W}$, we can see that $\rho_{2}$ is entangled for $p \leq 0.356$.

$$
\sqrt{F_{\rho}^{12 \cdots N-1}} \sqrt{\sum_{i} \Delta\left(A_{N}^{i}\right)^{2}-U_{N}} \pm \sum_{i}\left[\left\langle\left(A_{1}^{i}+\cdots+A_{N-1}^{i}\right) \otimes A_{N}^{i}\right\rangle-\left\langle A_{1}^{i}+\cdots+A_{N-1}^{i}\right\rangle\left\langle A_{N}^{i}\right\rangle\right] \geq 0,
$$

and

$$
\sqrt{F_{\rho}^{k_{0}}} \sqrt{F_{\rho}^{k_{1}}} \pm \sum_{i}\left[\left\langle\left(A_{1}^{i}+\cdots+A_{K}^{i}\right) \otimes\left(A_{K+1}^{i}+\cdots+A_{N}^{i}\right)\right\rangle-\left\langle A_{1}^{i}+\cdots+A_{K}^{i}\right\rangle\left\langle A_{K+1}^{i}+\cdots+A_{N}^{i}\right\rangle\right] \geq 0,
$$

where $\quad F_{\rho}^{12 \ldots N-1}=\sum_{i} \Delta\left(A_{1}^{i}+A_{2}^{i}+\cdots+A_{N-1}^{i}\right)^{2}-\left(\sum_{j=1}^{N-1} U_{j}+M_{12}^{2}+M_{12 \mid 3}^{2}+\cdots+M_{12 \cdots N-2 \mid N-1}^{2}\right)$, $F_{\rho}^{k_{0}}=\sum_{i} \Delta\left(A_{1}^{i}+A_{2}^{i}+\cdots+A_{K}^{i}\right)^{2}-\left(\sum_{j=1}^{K} U_{j}+M_{12}^{2}+M_{12 \mid 3}^{2}+\cdots+M_{12 \cdots K-1 \mid K}^{2}\right), F_{\rho}^{k_{1}}=\sum_{i} \Delta\left(A_{K+1}^{i}+\cdots+\right.$ $\left.A_{N}^{i}\right)^{2}-\left(\sum_{j=K+1}^{N} U_{j}+M_{K+1 K+2}^{2}+\cdots+M_{K+1 K+2 \cdots N-1 \mid N}^{2}\right)$.

Theorem 2 For any multipartite separable states, the following inequalities hold under any permutations of the subsystems,

$$
F_{\rho}^{k_{1} \mid k_{0}}=F-\left(M_{i_{1} i_{2}}^{2}+M_{i_{1} i_{2} i_{3}}^{2}+\cdots+M_{i_{1} i_{2} \cdots i_{K}}^{2}+M_{i_{K+1} i_{K+2}}^{2}+\cdots+M_{i_{K+1} i_{K+2} \cdots i_{N}}^{2}+M_{i_{1} i_{2} \cdots i_{N}}^{2}\right) \geq 0,
$$

where 


$$
F=\sum_{i=1}^{N} \Delta\left(A_{1}^{i}+A_{2}^{i}+\cdots+A_{N}^{i}\right)_{\rho}^{2}-\sum_{j=1}^{N} U_{j}
$$

and

$$
\begin{aligned}
& M_{k_{1} \mid k_{0}}=\sqrt{F_{\rho}^{k_{1} \mid k_{0}}}-\sqrt{\sum_{i} \Delta\left(A_{i_{N}}^{i}\right)^{2}-U_{i_{N}}} \text {, for } K=N-1, \\
& M_{k_{1} \mid k_{0}}=\sqrt{F_{\rho}^{k_{1}}}-\sqrt{F_{\rho}^{k_{0}}}, \text { for } K<N-1 .
\end{aligned}
$$

$A_{i_{1}}^{i}$ is an operator acting on the $i_{1}$-th subsystem $\mathcal{H}_{i_{1}}$ with the rest subsystems as identity operators in $N$-partite quantum systems.

Let us consider five-partite quantum systems to illustrate the theorem. In the case of $N=5$, we can have

$$
\left\{\begin{array}{l}
k_{1} \in\{123,124,125,134,135,145,234,235,245,345\} \text { and } k_{0} \in\{45,35,34,25,24,23,15,14,13,12\} K=3 ; \\
k_{1} \in\{1234,1235,1245,1345,2345\} \text { and } k_{0} \in\{5,4,3,2,1\}
\end{array}\right.
$$

Hence we have

$$
\begin{aligned}
& F_{\rho}^{1234 \mid 5}=F-\left(M_{12}^{2}+M_{12 \mid 3}^{2}+M_{123 \mid 4}^{2}\left(M_{12 \mid 34}^{2}\right)+M_{1234 \mid 5}^{2}\right), \\
& F_{\rho}^{1235 \mid 4}=F-\left(M_{12}^{2}+M_{12 \mid 3}^{2}+M_{123 \mid 5}^{2}\left(M_{12 \mid 35}^{2}\right)+M_{1235 \mid 4}^{2}\right), \\
& F_{\rho}^{1345 \mid 2}=F-\left(M_{13}^{2}+M_{13 \mid 4}^{2}+M_{134 \mid 5}^{2}\left(M_{13 \mid 45}^{2}\right)+M_{1345 \mid 2}^{2}\right), \\
& F_{\rho}^{2345 \mid 1}=F-\left(M_{23}^{2}+M_{23 \mid 4}^{2}+M_{234 \mid 5}^{2}\left(M_{23 \mid 45}^{2}\right)+M_{2345 \mid 1}^{2}\right), \\
& F_{\rho}^{1245 \mid 3}=F-\left(M_{12}^{2}+M_{12 \mid 3}^{2}+M_{124 \mid 5}^{2}\left(M_{12 \mid 45}^{2}\right)+M_{1245 \mid 3}^{2}\right), \\
& F_{\rho}^{123 \mid 45}=F-\left(M_{12}^{2}+M_{12 \mid 3}^{2}+M_{45}^{2}+M_{123 \mid 45}^{2}\right), F_{\rho}^{124 \mid 53}=F-\left(M_{12}^{2}+M_{12 \mid 4}^{2}+M_{53}^{2}+M_{124 \mid 53}^{2}\right), \\
& F_{\rho}^{125 \mid 34}=F-\left(M_{12}^{2}+M_{12 \mid 5}^{2}+M_{34}^{2}+M_{125 \mid 34}^{2}\right), F_{\rho}^{134 \mid 52}=F-\left(M_{13}^{2}+M_{13 \mid 4}^{2}+M_{52}^{2}+M_{134 \mid 52}^{2}\right), \\
& F_{\rho}^{135 \mid 24}=F-\left(M_{13}^{2}+M_{13 \mid 5}^{2}+M_{24}^{2}+M_{135 \mid 24}^{2}\right), F_{\rho}^{145 \mid 23}=F-\left(M_{14}^{2}+M_{14 \mid 5}^{2}+M_{23}^{2}+M_{145 \mid 23}^{2}\right), \\
& F_{\rho}^{234 \mid 51}=F-\left(M_{23}^{2}+M_{23 \mid 4}^{2}+M_{51}^{2}+M_{234 \mid 51}^{2}\right), F_{\rho}^{235 \mid 41}=F-\left(M_{23}^{2}+M_{23 \mid 5}^{2}+M_{41}^{2}+M_{235 \mid 41}^{2}\right), \\
& F_{\rho}^{245 \mid 13}=F-\left(M_{24}^{2}+M_{24 \mid 5}^{2}+M_{13}^{2}+M_{245 \mid 13}^{2}\right), F_{\rho}^{345 \mid 12}=F-\left(M_{34}^{2}+M_{34 \mid 5}^{2}+M_{12}^{2}+M_{345 \mid 12}^{2}\right),
\end{aligned}
$$

w h e r e

$$
F=\sum_{i} \Delta\left(A_{1}^{i}+A_{2}^{i}+\cdots+A_{5}^{i}\right)_{\rho}^{2}-\sum_{j=1}^{5} U_{j}, \quad M_{1234 \mid 5}=\sqrt{F_{\rho}^{123 \mid 4}}-\sqrt{\sum_{i} \Delta\left(A_{5}^{i}\right)^{2}-U_{5}},
$$

$M_{123 \mid 45}=\sqrt{F_{\rho}^{12 \mid 3}}-\sqrt{F_{\rho}^{45}} \cdot M_{2345 \mid 1}, M_{1345 \mid 2}, M_{1245 \mid 3}, M_{1235 \mid 4}, M_{124 \mid 53}, M_{125 \mid 34}, M_{134 \mid 52}, M_{135 \mid 24}, M_{145 \mid 23}, M_{234 \mid 51}$, $M_{235 \mid 41}, M_{245 \mid 13}, M_{345 \mid 12}$ have similar representations.

As a simple example, consider the five-qubit state $\rho=\left|W_{5}\right\rangle\left\langle W_{5}\right|$, with $\left|W_{5}\right\rangle=\frac{1}{\sqrt{5}}(|10000\rangle+|01000\rangle+|00100\rangle$ $+|00010\rangle+|00001\rangle)$. Let $-A_{1}^{1}=A_{2}^{1}=-A_{3}^{1}=-A_{4}^{1}=A_{5}^{1}=\sigma_{x}, \quad-A_{1}^{2}=-A_{2}^{2}=-A_{3}^{2}=A_{4}^{2}=A_{5}^{2}=\sigma_{y}$, $A_{1}^{3}=-A_{2}^{3}=-A_{3}^{3}=A_{4}^{3}=A_{5}^{3}=\sigma_{z}$. We have $U_{1}=U_{2}=U_{3}=U_{4}=U_{5}=2, M_{12}=M_{34}=0, M_{123}=0.2161$, $M_{123 \mid 4}=1.218, \quad M_{12 \mid 34}=0, \quad M_{1234 \mid 5}=0.2797$ and $M_{123 \mid 45}=0.8536$, which give rise to $F_{\rho}^{1234 \mid 5}=3-M_{123}^{2}-M_{1234}^{2}-M_{1234 \mid 5}^{2}<0$ and $F_{\rho}^{123 \mid 45}<0$, namely, the state is entangled.

\section{Conclusion}

We have generalized the LUR criterion for three qubit quantum systems to multiqubit quantum systems, and obtained new entanglement criteria for four-partite quantum systems as well as for general multipartite systems. By detailed examples we have shown that our criteria can detect better the entanglement than some existing criteria. It is further known that in certain situations they can provide a nonlinear refinement of linear entanglement witnesses ${ }^{35}$, and it can be measured in experimental settings similar to those of entanglement witnesses. The effectiveness of the LUR criteria relies heavily on certain notions of information content of quantum states and choice of observables.

Quantum entanglement is fundamentally connected to the quantum steering, local uncertainty relations (LURs) are a common tool for entanglement detection, and the underlying idea can be directly generalized to steering detection ${ }^{36}$.

The considered system here is closed systems with no decoherence effects taken into account. Also, it would be interesting to find criteria for open quantum systems, since realistic quantum systems inevitably interact with the environment. It would be also interesting if our approach may highlight further investigations on the $k$-separability ${ }^{37}$ of multipartite systems and genuine multipartite entanglement detection. 


\section{Methods}

Proof of the Theorem 1 By straightforward computation, we have

$$
\begin{aligned}
& \sum_{i} \Delta\left(A_{1}^{i}+A_{2}^{i}+A_{3}^{i}+A_{4}^{i}\right)_{\rho}^{2}=\sum_{i} \Delta\left(A_{1}^{i}+A_{2}^{i}+A_{3}^{i}\right)^{2}+\sum_{i} \Delta\left(A_{4}^{i}\right)^{2} \\
& \quad+2 \sum_{i}\left[\left\langle\left(A_{1}^{i}+A_{2}^{i}+A_{3}^{i}\right) \otimes A_{4}^{i}\right\rangle-\left\langle A_{1}^{i}+A_{2}^{i}+A_{3}^{i}\right\rangle\left\langle A_{4}^{i}\right\rangle\right] .
\end{aligned}
$$

Taking into account that for any tripartite separable states $\rho \in \mathcal{H}_{1} \otimes \mathcal{H}_{2} \otimes \mathcal{H}_{3}{ }^{33}$,

$$
\sqrt{F_{\rho}^{12}} \sqrt{\sum_{i} \Delta\left(A_{3}^{i}\right)^{2}-U_{3}} \pm \sum_{i}\left[\left\langle\left(A_{1}^{i}+A_{2}^{i}\right) \otimes A_{3}^{i}\right\rangle-\left\langle A_{1}^{i}+A_{2}^{i}\right\rangle\left\langle A_{3}^{i}\right\rangle\right] \geq 0,
$$

where $F_{\rho}^{12}=\sum_{i} \Delta\left(A_{1}^{i}+A_{2}^{i}\right)^{2}-\left(U_{1}+U_{2}+M_{12}^{2}\right)$, we obtain

$$
\sum_{i} \Delta\left(A_{1}^{i}+A_{2}^{i}+A_{3}^{i}+A_{4}^{i}\right)_{\rho}^{2} \geq U_{1}+U_{2}+U_{3}+U_{4}+M_{12}^{2}+M_{12 \mid 3}^{2}+M_{123 \mid 4}^{2}
$$

namely, $F_{\rho}^{123 \mid 4} \geq 0$. By relabeling the sub-indices, we have $F_{\rho}^{124 \mid 3} \geq 0, F_{\rho}^{134 \mid 2} \geq 0$ and $F_{\rho}^{234 \mid 1} \geq 0$, similarly. Concerning $F_{\rho}^{12 \mid 34}$, we have

$$
\begin{aligned}
& \sum_{i} \Delta\left(A_{1}^{i}+A_{2}^{i}+A_{3}^{i}+A_{4}^{i}\right)_{\rho}^{2}=\sum_{i} \Delta\left(A_{1}^{i}+A_{2}^{i}\right)^{2}+\sum_{i} \Delta\left(A_{3}^{i}+A_{4}^{i}\right)^{2} \\
& \quad+2 \sum_{i}\left[\left\langle\left(A_{1}^{i}+A_{2}^{i}\right) \otimes\left(A_{3}^{i}+A_{4}^{i}\right)\right\rangle-\left\langle A_{1}^{i}+A_{2}^{i}\right\rangle\left\langle A_{3}^{i}+A_{4}^{i}\right\rangle\right] .
\end{aligned}
$$

Since for any bipartite separable states $\rho \in \mathcal{H}_{1} \otimes \mathcal{H}_{2}$, the following inequality holds ${ }^{33}$,

$$
\sqrt{\sum_{i} \Delta\left(A_{2}^{i}\right)^{2}-U_{1}} \sqrt{\sum_{i} \Delta\left(A_{2}^{i}\right)^{2}-U_{2}} \pm \sum_{i}\left[\left\langle A_{1}^{i} \otimes A_{2}^{i}\right\rangle-\left\langle A_{1}^{i}\right\rangle\left\langle A_{2}^{i}\right\rangle\right] \geq 0,
$$

we get

$$
\sum_{i} \Delta\left(A_{1}^{i}+A_{2}^{i}+A_{3}^{i}+A_{4}^{i}\right)_{\rho}^{2} \geq U_{1}+U_{2}+U_{3}+U_{4}+M_{12}^{2}+M_{34}^{2}+M_{12 \mid 34}^{2}
$$

namely, $F_{\rho}^{12 \mid 34} \geq 0$. Similarly one can show that $F_{\rho}^{23 \mid 41} \geq 0$ and $F_{\rho}^{13 \mid 42} \geq 0$.

Proof of the Theorem 2 We denote the length of $k_{0}$ as $\left|k_{0}\right|$. From above, one has $\left|k_{0}\right|+\left|k_{1}\right|=N$.

When $K=N-1$, one has $\left|k_{0}\right|=1$, by straightforward computation, we have

$$
\begin{aligned}
& \sum_{i} \Delta\left(A_{1}^{i}+A_{2}^{i}+\cdots+A_{N}^{i}\right)_{\rho}^{2}=\sum_{i} \Delta\left(A_{1}^{i}+A_{2}^{i}+\cdots+A_{N-1}^{i}\right)^{2}+\sum_{i} \Delta\left(A_{N}^{i}\right)^{2} \\
& \quad+2 \sum_{i}\left[\left\langle\left(A_{1}^{i}+A_{2}^{i}+\cdots+A_{N-1}^{i}\right) \otimes A_{N}^{i}\right\rangle-\left\langle A_{1}^{i}+A_{2}^{i}+\cdots+A_{N-1}^{i}\right\rangle\left\langle A_{N}^{i}\right\rangle\right] .
\end{aligned}
$$

By Lemma 2, for any multipartite separable states $\rho \in \mathcal{H}_{1} \otimes \mathcal{H}_{2} \otimes \cdots \otimes \mathcal{H}_{N}$,

$$
\begin{aligned}
& \sqrt{F_{\rho}^{12 \cdots N-1}} \sqrt{\sum_{i} \Delta\left(A_{N}^{i}\right)^{2}-U_{N}} \\
& \quad \pm \sum_{i}\left[\left\langle\left(A_{1}^{i}+A_{2}^{i}+\cdots+A_{N-1}^{i}\right) \otimes A_{N}^{i}\right\rangle-\left\langle A_{1}^{i}+A_{2}^{i}+\cdots+A_{N-1}^{i}\right\rangle\left\langle A_{N}^{i}\right\rangle\right] \geq 0,
\end{aligned}
$$

via calculation, we obtain

$$
\sum_{i} \Delta\left(A_{1}^{i}+A_{2}^{i}+\cdots+A_{N}^{i}\right)_{\rho}^{2} \geq \sum_{j=1}^{N} U_{j}+M_{12}^{2}+M_{12 \mid 3}^{2}+\cdots+M_{12 \cdots N-1 \mid N}^{2},
$$

namely, $F_{\rho}^{12 \cdots N-1 \mid N} \geq 0$. By relabeling the sub-indices, we have $F_{\rho}^{k_{1} \mid k_{0}} \geq 0$.

When $K<N-1$, one has $\left|k_{0}\right| \geq 2$, 


$$
\begin{aligned}
\sum_{i} \Delta\left(A_{1}^{i}+\cdots+A_{N}^{i}\right)_{\rho}^{2}= & \sum_{i} \Delta\left(A_{1}^{i}+\cdots+A_{K}^{i}\right)^{2}+\sum_{i} \Delta\left(A_{K+1}^{i}+\cdots+A_{N}^{i}\right)^{2} \\
& +2 \sum_{i}\left[\left\langle\left(A_{1}^{i}+\cdots+A_{K}^{i}\right) \otimes\left(A_{K+1}^{i}+\cdots+A_{N}^{i}\right)\right\rangle-\left\langle A_{1}^{i}+\cdots+A_{K}^{i}\right\rangle\left\langle A_{K+1}^{i}+\cdots+A_{N}^{i}\right\rangle\right] .
\end{aligned}
$$

By using Lemma 2, we get

$$
\sum_{i} \Delta\left(A_{1}^{i}+A_{2}^{i}+\cdots+A_{N}^{i}\right)_{\rho}^{2} \geq \sum_{j=1}^{N} U_{j}+\left(M_{12}^{2}+M_{12 \mid 3}^{2}+\cdots+M_{12 \cdots \mid K}^{2}+M_{K+1 K+2}^{2}+\cdots+M_{K+1 \cdots \mid N}^{2}+M_{12 \cdots \mid N}^{2}\right),
$$

namely, $F_{\rho}^{12 \cdots K \mid K+1 K+2 \cdots N} \geq 0$. By relabeling the sub-indices, one can show that $F_{\rho}^{k_{0} \mid k_{1}} \geq 0$.

Received: 5 February 2021; Accepted: 12 April 2021

Published online: 05 May 2021

\section{References}

1. Nielsen, M. A. \& Chuang, I. L. Quantum Computation and Quantum Information Anniversary (Cambridge University Press, 2010).

2. Leibfried, D. et al. Creation of a six-atom 'Schrödinger cat' state. Nature (London) 438, 639 (2005).

3. Lu, C. Y. et al. Experimental entanglement of six photons in graph states. Nat. Phys. 3, 91 (2007).

4. Verstraete, F. \& Verschelde, H. Optimal teleportation with a mixed state of two qubits. Phys. Rev. Lett. 90, 097901 (2003).

5. Lee, S., Joo, J. \& Kim, J. Teleportation capability, distillability, and nonlocality on three-qubit states. Phys. Rev. A 76, 012311 (2007).

6. Cleve, R., Gottesman, D. \& Lo, H. K. How to share a quantum secret. Phys. Rev. Lett. 83, 648 (1999).

7. Rab, A. S. et al. Entanglement of photons in their dual wave-particle nature. Nat. Commun. 8, 915 (2017).

8. Werner, R. F. Quantum states with Einstein-Podolsky-Rosen correlations admitting a hidden-variable model. Phys. Rev. A 40, 4277 (1989).

9. Liu, B., Li, J. L., Li, X. \& Qiao, C. F. Local unitary classification of arbitrary dimensional multipartite pure states. Phys. Rev. Lett. 108, 050501 (2012).

10. Gour, G. \& Wallach, N. R. Classification of multipartite entanglement of all finite dimensionality. Phys. Rev. Lett. 111, 060502 (2013).

11. Hofmann, H. F. \& Takeuchi, S. Violation of local uncertainty relations as a signature of entanglement. Phys. Rev. A 68, 032103 (2003).

12. Hofmann, H. F. Bound entangled states violate a nonsymmetric local uncertainty relation. Phys. Rev. A 68, 034307 (2003).

13. Gühne, O., Hyllus, P., Gittsovich, O. \& Eisert, J. Covariance matrices and the separability problem. Phys. Rev. Lett. 99, 130504 (2007).

14. Chen, K. \& Wu, L. A. A matrix realignment method for recognizing entanglement. Quan. Inf. Comput. 3, 193 (2003).

15. Horodecki, M., Horodecki, P. \& Horodecki, R. Separability of mixed quantum states: linear contractions and permutation criteria. Open Syst. Inf. Dyn. 13, 103 (2006).

16. Horodecki, M., Horodecki, P. \& Horodecki, R. Separablity of mixed stated states: Necessary and sufficient conditions. Phys. Lett. A 223, 1 (1996).

17. de Julio, I. V. Separability criteria based on the Bloch representation of density matrices. Quan. Inf. Comput. 7, 624 (2007).

18. de Julio, I. V. Further results on entanglement detection and quantification from the correlation matrix criterion. J. Phys. A 41, 065309 (2008).

19. Fei, S. M., Zhao, M. J., Chen, K. \& Wang, Z. X. Experimental determination of entanglement for arbitrary pure states. Phys. Rev. A 80, 032320 (2009).

20. Peres, A. Separability criterion for density matrices. Phys. Rev. Lett. 77, 1413 (1996).

21. Gühne, O. \& Lütkenhaus, N. Nonlinear entanglement witnesses. Phys. Rev. Lett. 96, 170502 (2006).

22. Laskowski, W. \& Zukowski, M. Detection of N-particle entanglement with generalized Bell inequalities. Phys. Rev. A 72, 062112 (2005).

23. Kourbolagh, Y. A. \& Azhdargalam, M. Entanglement criterion for multipartite systems based on quantum Fisher information. Phys. Rev. A 99, 12304 (2019).

24. Briegel, H. J., Browne, D. E., Dür, W., Raussendorf, R. \& Van den Nest, M. Measurement-based quantum computation. Nat. Phys. 5, 19 (2009).

25. Amico, L., Fazio, R., Osterloh, A. \& Vedral, V. Entanglement in many-body systems. Rev. Mod. Phys. 80, 517 (2008).

26. Horodecki, R., Horodecki, P., Horodecki, M. \& Horodecki, K. Quantum entanglement. Rev. Mod. Phys. 81, 865 (2009).

27. Gühne, O. \& Tóth, G. Entanglement detection. Phys. Rep. 474, 1 (2009).

28. Shi, Y., Duan, L. \& Vidal, G. Classical simulation of quantum many-body systems with a tree tensor network. Phys. Rev. A 74, 022320 (2006).

29. Li, M., Fei, S. M. \& Wang, Z. X. Separability and entanglement of quantum states based on covariance matrices. J. Phys. A 41, 202002 (2008).

30. Zhang, C. J., Zhang, Y. S., Zhang, S. \& Guo, G. C. Entanglement detection beyond the computable cross-norm or realignment criterion. Phys. Rev. A 77, 060301(R) (2008).

31. Zhang, C. J., Nha, H., Zhang, Y. S. \& Guo, G. C. Entanglement detection via tighter local uncertainty relations. Phys. Rev. A 81, $012324(2010)$.

32. Zhao, M. J., Wang, Z. X. \& Fei, S. M. Multiqubits entanglement witness based on W state. Rep. Math. Phys. 63, 409 (2009).

33. Kourbolagh, Y. A. \& Azhdargalam, M. Entanglement criterion for tripartite systems based on local sum uncertainty relations. Phys. Rev. A 97, 042333 (2018).

34. Aulbach, M. Classification of entanglement in symmetric states. Int. J. Quantum Inf. 10, 1230004 (2012).

35. Gühne, O., Mechler, M. \& Peter Adam, G. T. Entanglement criteria based on local uncertainty relations are strictly stronger than the computable cross norm criterion. Phys. Rev. A 74, 010301(R) (2006).

36. Uola, R., Costa, A. C. S., Nguyen, H. C. \& Gühne, O. Quantum steering. Rev. Mod. Phys. 92, 015001 (2020)

37. Hong, Y. \& Luo, S. L. Detecting k-nonseparability via local uncertainty relations. Phys. Rev. A 93, 042310 (2016).

\section{Acknowledgements}

This work is supported by the Natural Science Foundation of China under Grant No. 11675113; Beijing Municipal Commission of Education (KZ201810028042); Beijing Natural Science Foundation (Grant No. Z190005); 
Academy for Multidisciplinary Studies, Capital Normal University; Shenzhen Institute for Quantum Science and Engineering, Southern University of Science and Technology, Shenzhen 518055, China (No. SIQSE202001).

\section{Author contributions}

The first and the second authors wrote the main manuscript text and all authors reviewed the manuscript.

\section{Competing interests}

The authors declare no competing interests.

\section{Additional information}

Correspondence and requests for materials should be addressed to T.L.

Reprints and permissions information is available at www.nature.com/reprints.

Publisher's note Springer Nature remains neutral with regard to jurisdictional claims in published maps and institutional affiliations.

(c) (i) Open Access This article is licensed under a Creative Commons Attribution 4.0 International License, which permits use, sharing, adaptation, distribution and reproduction in any medium or format, as long as you give appropriate credit to the original author(s) and the source, provide a link to the Creative Commons licence, and indicate if changes were made. The images or other third party material in this article are included in the article's Creative Commons licence, unless indicated otherwise in a credit line to the material. If material is not included in the article's Creative Commons licence and your intended use is not permitted by statutory regulation or exceeds the permitted use, you will need to obtain permission directly from the copyright holder. To view a copy of this licence, visit http://creativecommons.org/licenses/by/4.0/.

(C) The Author(s) 2021 